\title{
Aerodynamic simulation of windflow aroundurban regionsusing different turbulence modeling approaches
}

\author{
Shako A. Mohammed ${ }^{1 *}$ Saman A. Abdullah ${ }^{2}$ \\ ${ }^{1}$ College of Engineering, University of Sulaimani, Sulaymaniyah, Iraq \\ ${ }^{2}$ Civil and Environmental Engineering, University of California, Los Angeles, USA \\ *Corresponding author. Email: Shako.mohammed@univsul.edu.iq
}

\begin{abstract}
There are different turbulent models that have significant impacts on aerodynamic performance and simulation of wind flow over urban areas. A built-up urban area that contains a set of mid- to high-rise buildings was used to highlight theimpact of different turbulence models such asRe-Normalization Group(RNG)k-epsilon, ShearStress-Transport (SST)k-omega on the aerodynamic performance of wind flowingin different directions.For both approaches, simulation results such as pressure, mean velocity, and kinetic energy at different directions were obtained and compared. The results demonstrate that, givenan acceptable iterative time andthe same boundary conditions and input variables, both modeling approachesproduce similar resultsfor pressure and velocity around the urban area and slightly different results for the kinetic energy at some location in the urban region, as well as along the height of the buildings considered herein.
\end{abstract}

Keywords:Wind flow; winds pressure; wind velocity; Computational Fluid Dynamics (CFD); Turbulence models; $k-\varepsilon$ RANS model; $k-\omega$ SST model.

\section{Introduction}

Aerodynamic characteristics of buildings during wind events is one of the most important considerations in analysis and design of mid- and high-rise buildings because design of such structures is significantly impacted by wind-induced static and dynamic effects. Currently, understanding the true behaviour of buildings subjected to wind pressures as well as investigating the aeroelasticbehaviour of slender and lightweight buildings in urban regions can be cumbersome and expensive as it requires performing wind-tunnel testing procedures. An alternative approach to the time-consuming and costly wind-tunnel experiment is utilizing numerical based approaches of Computational Fluid Dynamics (CFD)(Wang et al., 2014), whichhas recently become the focus of research efforts concerned with aerodynamic controversial issues. CFD is a branch of fluid mechanics commonly used to solve and analyze complex scientific and engineering problemsthrough numerical analyses and 
algorithms, not only in fluid dynamics but also in other engineering disciplines such as aerodynamic, environmental, and thermodynamicengineering(H. Hu, 2012). Since methods used in CFD are very strict and complex, software such as Fluent, Star, and CFX and platforms to write CFD codes such as OpenFoam are available to aid for analysis. On the other hand, CFD method can be significantly used for solving the Navier Stokes equations (Lee D., 1993).Although, the existing world-leading standards and specifications in the field of aerodynamic engineering (e.g. ASCE, 2013)are using some experimental approaches in dealing with the various impacts of wind on buildings, the wind-tunnel procedure is utilized as a pre-design step for many types of buildings, especially for high rise buildings and those that have irregularities in geometry and/or function. Thus, the numerical turbulence models can be seen as a remarkablealternative to the experimental approaches (Wang et al., 2014).

Reviewing the available literature reveals(e.g. Ping He et al., 1997; A.K. Roy and P.K. Bhargava, 2012 and many others) that most studieshave applied only one turbulence model to simulate the aerodynamic performance of wind flow over a terrain, which can produce inaccurate results(Wang et al., 2014). Therefore, two different turbulence modeling approaches (i.e., RNG k-epsilonandSST k-omega) were studied herein and the results obtained for each approach (such as pressure, velocity, and kinematic energy)were compared for wind blowing in both orthogonal directions.Based on the results of the study, it can be concluded that utilizing more than one turbulence model can effectively increase the reliability of the achieved simulation results.

\section{Turbulence Models}

The wind flow around urban regions is dealt to have a constant density over the pathway and also belongs to the low flow problems. Hence, the wind can behave as an incompressible fluid in its simulation and/or calculation process. As a result, solving the energy equation is notthe point of interest because consideration of effects of heat transfer in the simulation process is not needed.On the other hand, RANS models calculate the equations of transport only for an average amount of the airflow; therefore, the results are not accurate (Reiter, 2008). Furthermore, the most general and simple model in the RANS approaches is the standard k-epsilon model, which has been employed by many researchers. Alternatively, there are some other reliable modeling approaches in RANS that work more accurately compared to the simple standard k-epsilon model, e.g.,RNG k-epsilondeveloped by Yakhot et al. (1992) andSST k-omegadeveloped by Menter(1993).This studyattempts to utilizeReynolds- 
averaged viscous incompressible Navier-Stokes equationsin OpenFOAM toolbox software for both models. Details of each modeling approach are presented next.

\subsection{RNG k-epsilon turbulence model}

RNG k-epsilon turbulence model is basically an improved version of the standard kepsilon model with some enhancements to increase the accuracy of the results. It was validated for a wide range of Reynold's number of fluids by providing an analytical formula for turbulent number Prandtl which allows for using a user-supplied constant(Wang et al., 2014). These features make the RNG k-epsilon a more reliable and efficient modelin dealing with wider engineering circumstances than the classic standard k-epsilon approach. Numerical equations of the RNG k-epsilon model are given in Eq. (1) and Eq. (2) as follows:

$$
\begin{aligned}
& \frac{D k}{D t}=\frac{\partial}{\partial x_{i}}\left[\left(\begin{array}{ll}
k & \text { eff }
\end{array}\right) \frac{\partial k}{\partial x_{i}}\right]+G_{k}+G_{b} \quad Y_{M} \quad \text { Eq. (1) } \\
& \frac{D}{D t}=\frac{\partial}{\partial x_{i}}\left[\left(\quad e_{e f f}\right) \frac{\partial k}{\partial x_{i}}\right]+C_{1} \frac{-}{k}\left(G_{k}+C_{3} G_{b}\right) \quad C_{2} \stackrel{\frac{2}{k}}{R} \quad R \quad \text { Eq. (1) }
\end{aligned}
$$

Where, $Y_{M}$ isthe effects of the compressible turbulence expansion on the total dissipation rate, $G_{k}$ and $G_{b}$ are the turbulent kinetic energy generated by both average velocity gradient and buoyancy, respectively, $\alpha_{k}$ and $\alpha_{\varepsilon}$ are the inverse parameters for the turbulent Prandtl number in both the kinetic energy $\mathrm{k}$ and dissipation rate $\varepsilon$, respectively.

\subsection{SST k-omega turbulence model}

This model consists of the two-equation eddy-viscosity models, which are the Wilcox k-omega and the k-epsilon models used to simulate the transit of flow near the wall and remote boundary, respectively,(Wang et al., 2014). It also can be used as a low Reynold's turbulence model without adding any kind of dumping functions (Menter, 1993). Compared to the standard k-omega model, there are some improvements in the SST k-omega model such as adding a special cross-diffusion in the omega $(\omega)$ equation and utilizing constants in both models are different. Therefore, compared to the normal k-epsilon model, this model produces a larger turbulence levels, especially in regions of large normal strains. The flow equations of the SST k-omega model are given in Eq. (3) and Eq. (4) in the following:

$$
\frac{\partial}{\partial t}(k)+\frac{\partial}{\partial x_{i}}\left(k u_{i}\right)=\frac{\partial}{\partial x_{j}} \backslash \quad{ }_{k} \frac{\partial k}{\partial x_{j}} \mid+G_{k} \quad Y_{k}+S_{k}
$$




$$
\frac{\partial}{\partial t}(\quad)+\frac{\partial}{\partial x_{i}}\left(\quad u_{i}\right)=\frac{\partial}{\partial x_{j}} \square \quad \frac{\partial}{\partial x_{j}} \mid+G \quad Y+D+S
$$

Where $\Gamma_{k}$ and $\Gamma_{\omega}$ arethe effective diffusion terms of $k$ and $\omega$, respectively, $G_{k}$ and $G_{\omega}$ represent both the kinetic energy and omega equations, respectively, $D_{\omega}$ isthe orthogonal divergent term, and $S_{k}$ and $S_{\omega}$ are parameters defined by the user.

\section{Simulation Model}

\subsection{Model definition}

The idealized prototype urban area developed in this study contains many buildings with various heights ranging mid-rise to high-rise buildings(up to $100 \mathrm{~m}$ tall) and various shapes (i.e., regular and irregular cross-sectional shapes) spaced at various distances, as shown in Figure 1. This was to ensure that the prototype region used herein represents a somewhat realistic urban region.

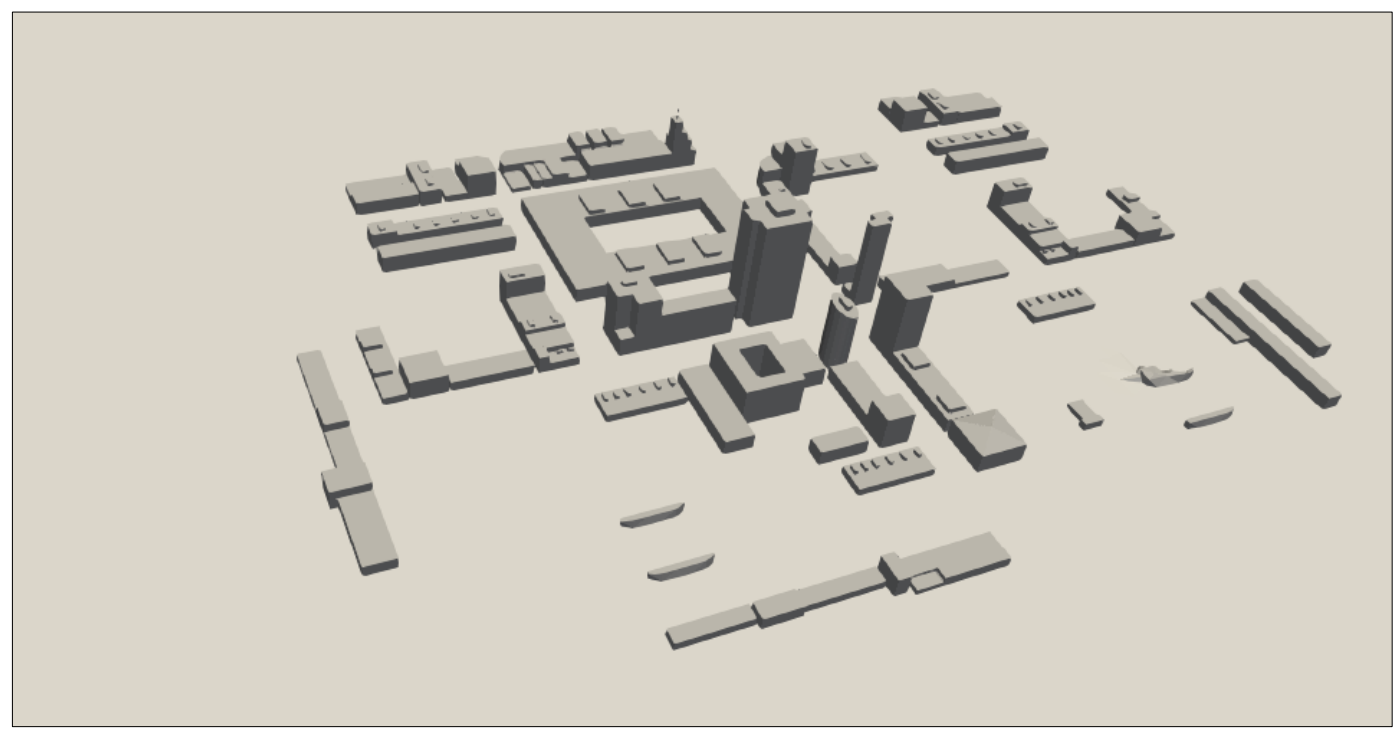

Figure 1: The prototype urban region used in this study.

\subsection{Mesh generation}

Mesh generation is a crucial step innumerical simulation of any engineering challenge because quality of the mesh can significantly impact the outcomes of the analyses. In this study, the geometry of the idealized urban area wasfirst developedin AutoCAD and was then exported into OpenFOAM simulation tool. Computational structured grid wasutilized to generate meshing of the buildings and surroundings in the form of snappyHexMesh(Figure 2). After that, the meshes were checked for uniformity, aspect ratio, orthogonality, and skewness because these mesh metrics would affect accuracy, robustness, and efficiency of the resulted mesh. As the snappyHexMesh isnot capable of creating sharp edges for the buildings, an approach using surfaceExtractDictfile was employed to generate sharp edges in the analysis. 

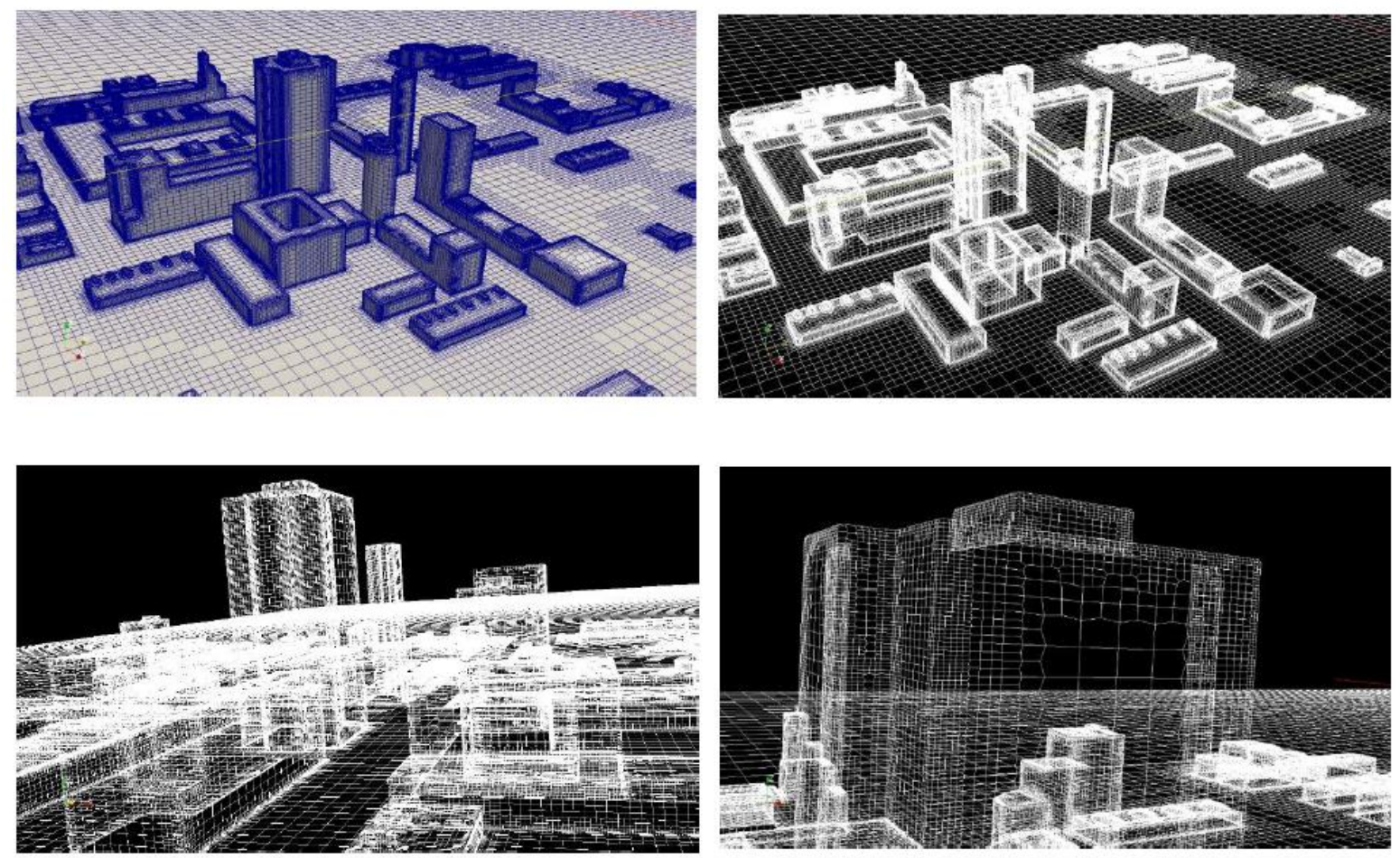

Figure 2: Mesh generation of the urban area by the snappyHexMesh.

\section{Domain, Boundary Conditions, and Initial Conditions}

Domain means a boundary region bounded by the targeted area, which can imitate the real environmental conditions for the region. In CFD, each issue has its own criteria for size and shape of its domain that are recommended by relevant standard codes or research studies. On the other hand, the urban area should have a domain that could represent the real condition of atmosphere and;

therefore, it must be large enough for simulating the wind flow. There are several recommendations suggested by researchers working in the areaof wind engineering (e.g., Hall, 1997). Depend on that, the domain should have an inlet layer and lateral sides away from the urban region by $5 \times H_{\max }$, where $H_{\max }$ is the height of the tallest buildingin the region. Furthermore, the out flow sides of the domain should be away from the area by $15 \times H_{\max }$. The top of the computational domain should also be away from the tallest building by $5 \times H_{\max }$. Figure 3 shows the recommended domain for the issue. In addition, each domain has some boundary faces bounded by the domain used to describe the inlet/outlet path and sides of the flow through the domain. 


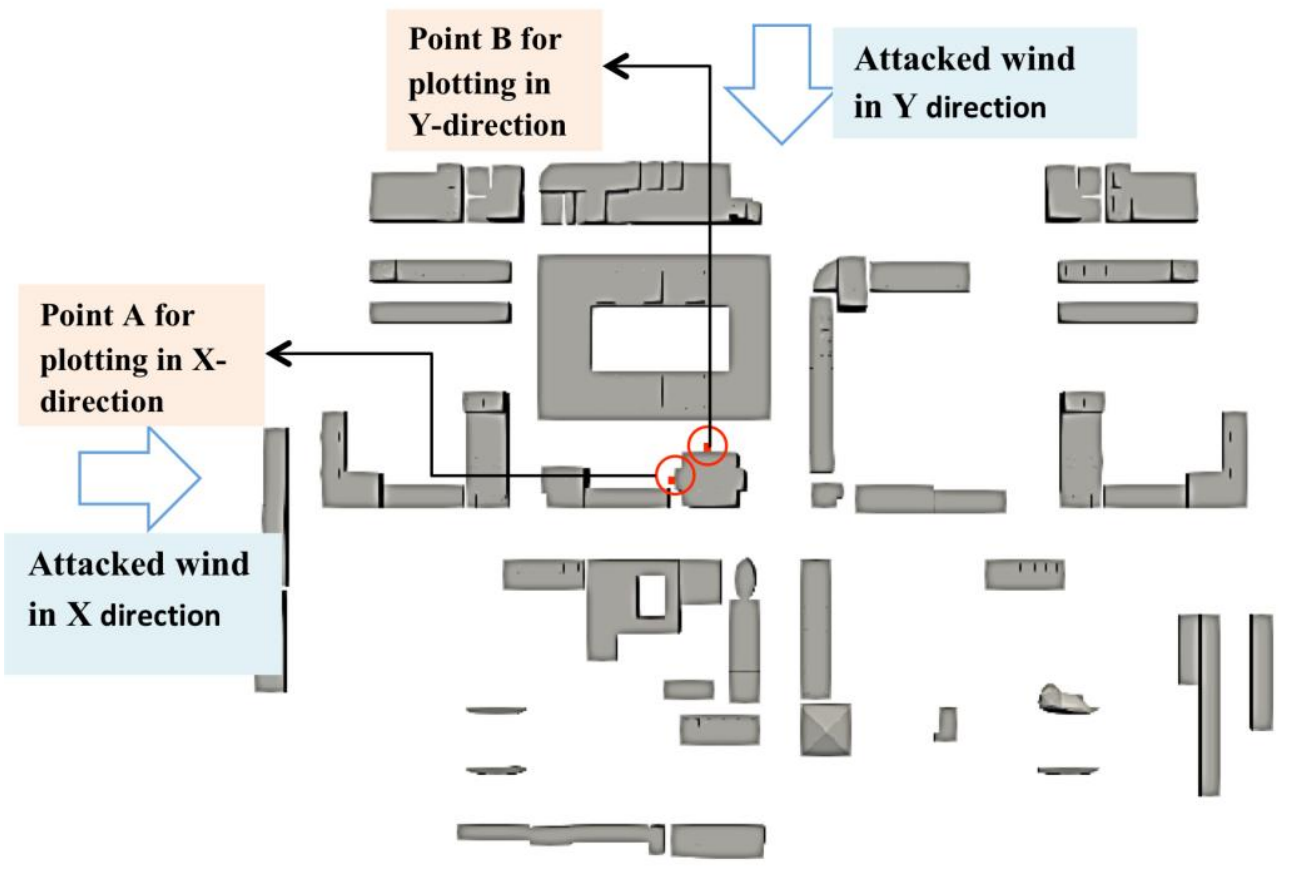

Figure 3: Top view of the urban area

In regards with the initial conditions for the wind flow, this study uses uniform profiles for the velocity and other parameters (e.g., pressure, Reynold number, kinematic energy, and epsilon). However, there is non-uniformity of these parameters in the atmosphere. That is, they have different valuesatdifferent heights above the ground. Thus, a monotonic magnitude of $40 \mathrm{~m} / \mathrm{sec}$ asemployed for the wind velocity as its initial inlet value into the domain. This leads to calculatingthe Reynold's number and turbulent intensity for the wind flow through the domain. Generally, the turbulent intensity has a value in the range of $1 \%$ to $20 \%$. For the wind flow, a small value of turbulent intensity has commonly, been used because Reynold's number of wind is much higher-in some complex situations it could be more than 1 billion. Furthermore, the turbulent intensity plays a major rolein figuring out the initial inlet values of kinetic energy and its dissipation rate. Table 1 shows the calculated values for all the requiredparameters used in the simulation.It is noted that these values are initial physical conditions of the wind flow, and that they would change at any iteration until the simulation reaches the pre-defined convergence value for each of them.

Table 1: Initial input values of theparameters.

\begin{tabular}{|c|c|c|c|c|}
\hline Parameter & $\begin{array}{c}\text { Mean velocity } \\
(\mathrm{m} / \mathrm{sec})\end{array}$ & $\begin{array}{c}\text { Kinetic energy, } \\
\mathrm{k}\left(\mathrm{m}^{2} / \mathrm{sec}^{2}\right)\end{array}$ & $\begin{array}{c}\text { Epsilon, } \varepsilon \\
\left(\mathrm{m}^{2} / \mathrm{sec}^{3}\right)\end{array}$ & $\begin{array}{c}\text { Omega, } \omega \\
(1 / \mathrm{sec})\end{array}$ \\
\hline Initial values & 40 & 0.00375 & 0.0125 & 3.375 \\
\hline
\end{tabular}




\section{Simulation and Results}

\subsection{Simulation conditions}

In order to perform an efficient comparison between RNG k-epsilon and k-omega turbulence models, the same boundary and initial conditions were set for the simulation. Linear SIMPLE algorithms were utilized for both models as the main solvers for the calculation process of velocity, and GAMG(generalized geometricalgebraic multi-grid) solver was used as a pressure solver, whereas Smooth solver was used for the other parameters. Pressure residual control was taken as 0.0001 and a value of 0.001 was set for the other variables in the simulation.Comparison between the two models was carried out in two steps. First, looking at different schemes of the simulated output results on the same sections and/or the same environmental conditions in the Paraview software. For this, a single section was chosen in each attacked wind direction. Second, variation of velocity, pressure, and the kinetic energy was plotted along the height of the building at a location on the face of the tallest building, for each orthogonal direction (i.e., $\mathrm{X}$ and $\mathrm{Y}$-directions), as it can be seen in Figure 3.

\subsection{Simulation and comparison of turbulence models}

Results of variation of velocity, pressure, and kinetic energy at the point A for Xdirection and point $\mathrm{B}$ for $\mathrm{Y}$-direction of wind flow are compared for both turbulence modeling approaches in Figure 4 and Figure 5, respectively. From these figures, it can clearly be seen that the predicted pressure and velocity profiles obtained from both modeling approaches are identical, whereas significant difference can be seen in the kinetic energy up to $40 \mathrm{~m}$ height; the k-epsilon model predicts higher kinetic energy than the k-omega model.

Figures $4 \mathrm{a}$ and $4 \mathrm{~b}$ show that the pressure profile is the reverse of the velocity profile, which indicates that when the wind flow attacks the face of the building, it creates pressure on that face and loses most of its energy; therefore, its speed magnitudefalls down. Another important aspect to be considered in this regard is creating the vortex shading in front of the point A that dissipates the magnitude of the velocity and also falls down the amount of kinetic energy on those zones.

Flow fields of the simulated results obtained from both turbulence models are shown in Figure 6 and Figure 7 for X-and Y-directions, respectively. From these figures, it can be observed that the flow fields turbulence models for both models are also identical for velocity and pressure, except for pressures on the roof of the tall building. However, there are still significant differences in the simulated results of kinetic energy obtained from the two modeling approaches. It can also be seen that 
there is higher kinetic energy produced around the buildings using the RNG k-epsilon model. Furthermore, compared to the SST k-omega model, the k-epsilon mdels exhibits a higher reduction rate along the height of the building.
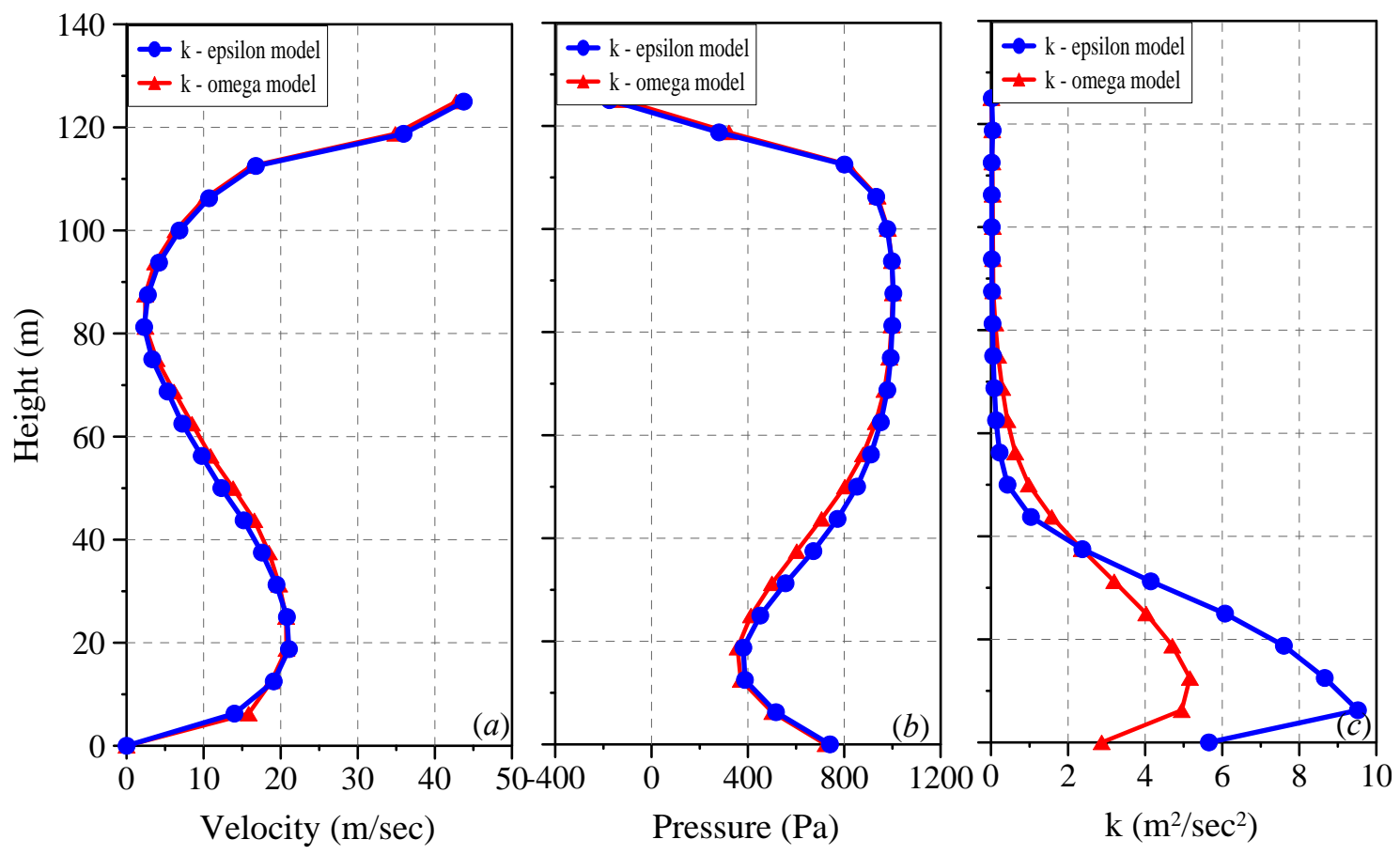

Figure 4: Profiles of (a) velocity, (b) pressure, and (c) kinetic energy at point A in the X-direction.
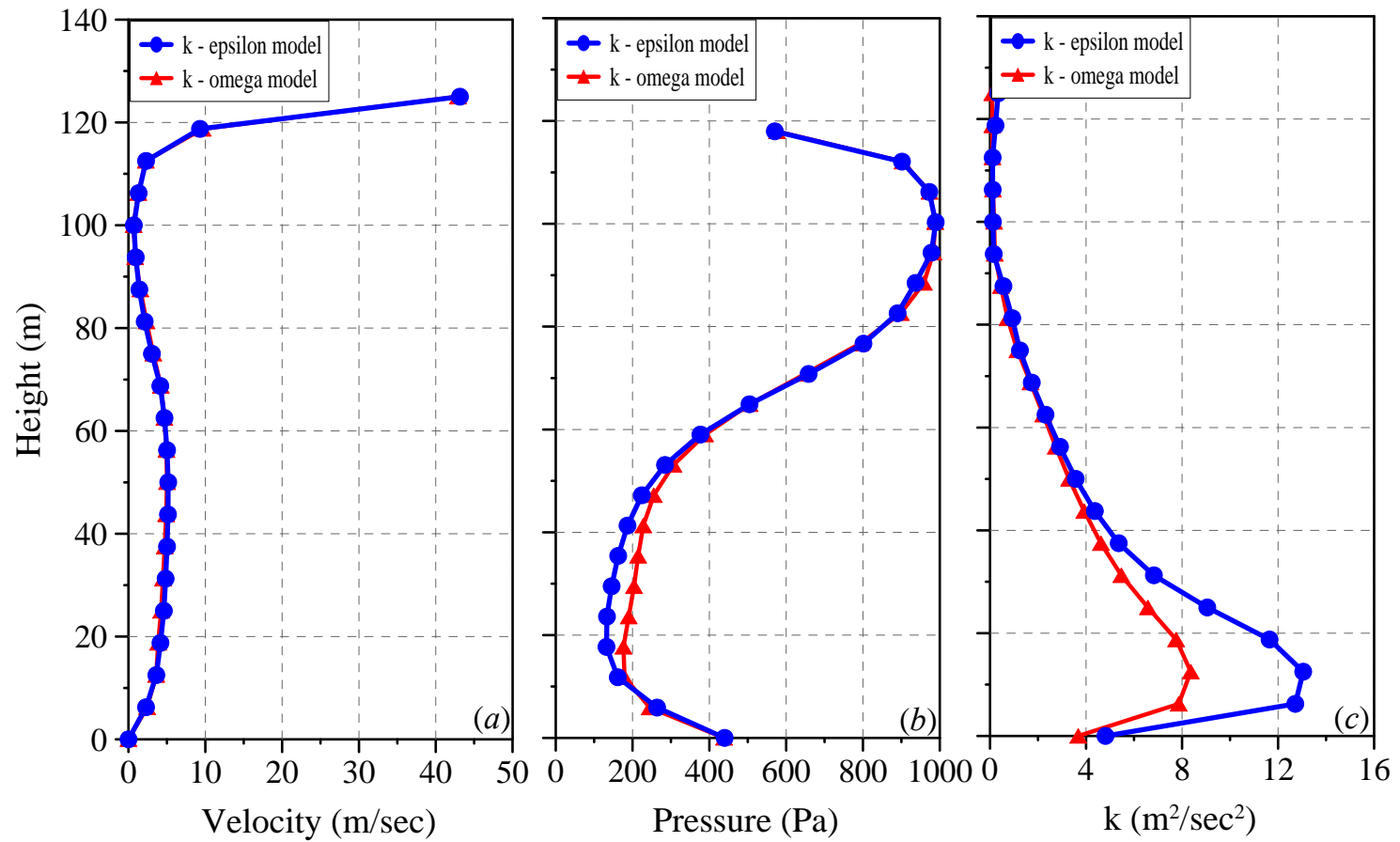

Figure 5: Profiles of (a) velocity, (b) pressure, and (c) kinetic energy at point B in the Y-direction. 

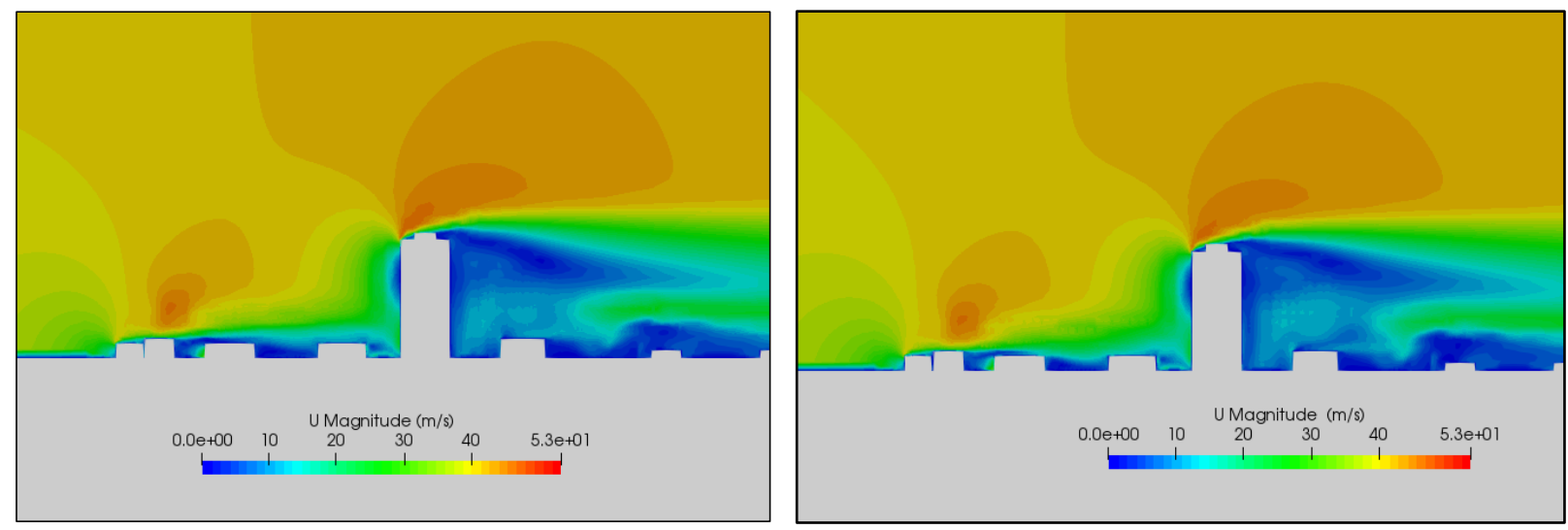

(a) Velocity

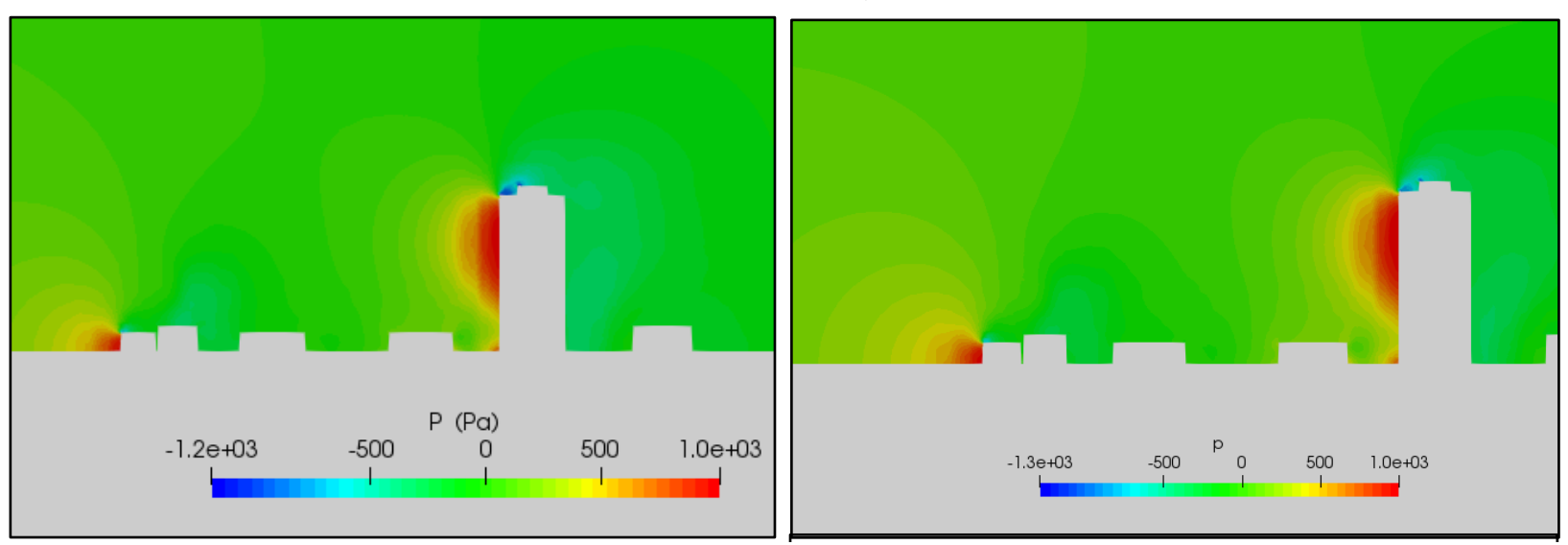

(b) Pressure
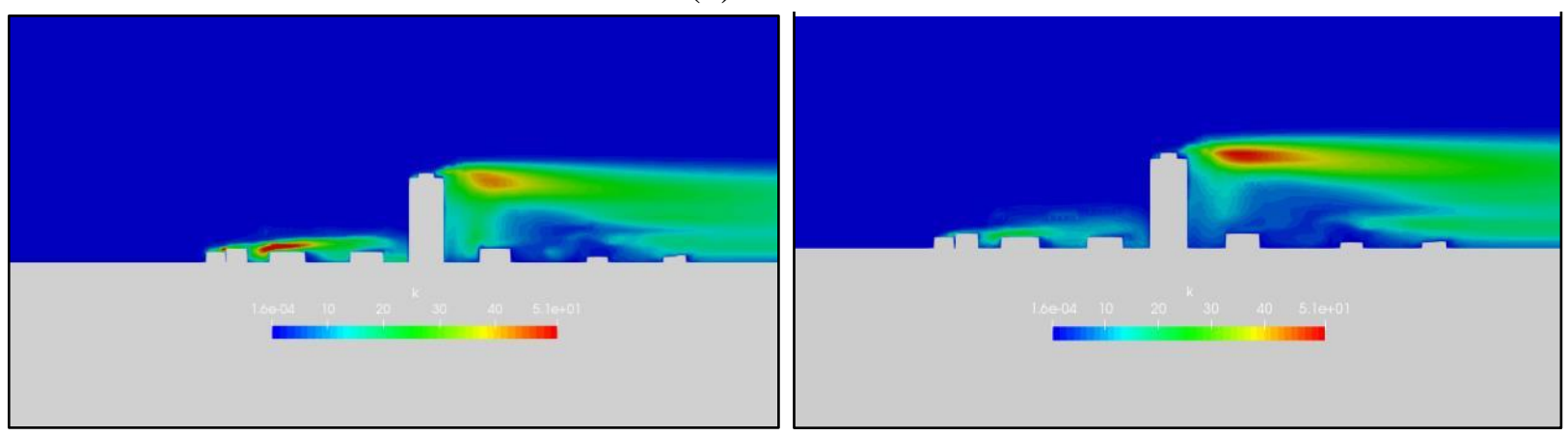

(c) Kinetic energy

Figure 6: The comparison of flow field in X-direction for both RNG k-epsilon model (left side) and SST k-omega model (right side).
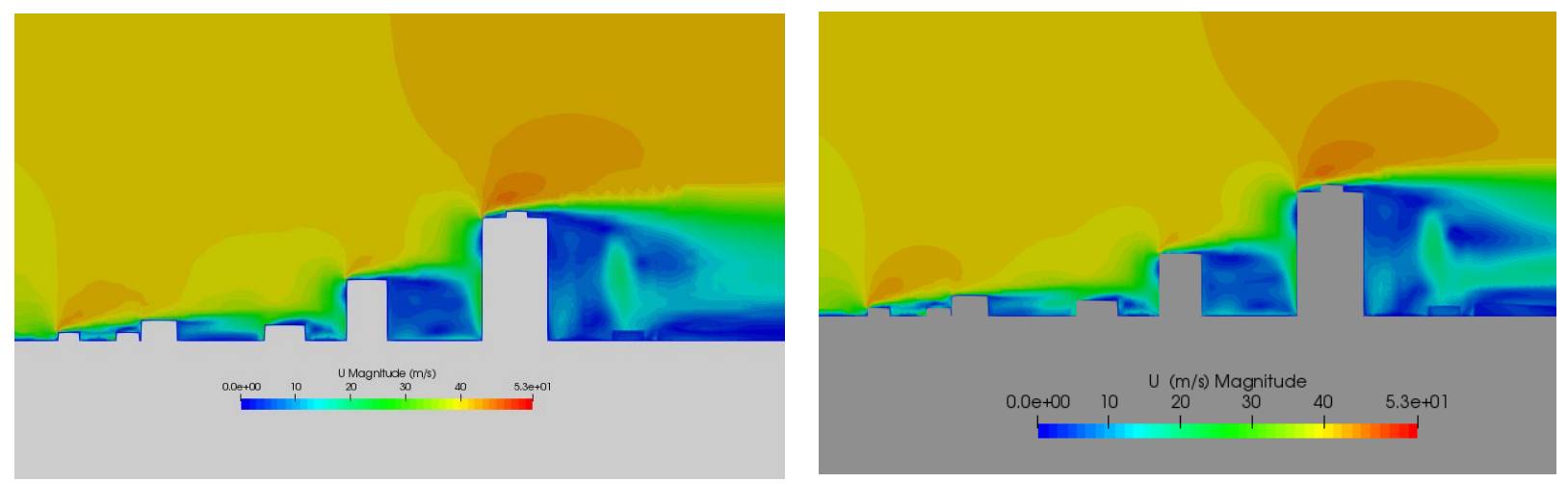
(a) Velocity
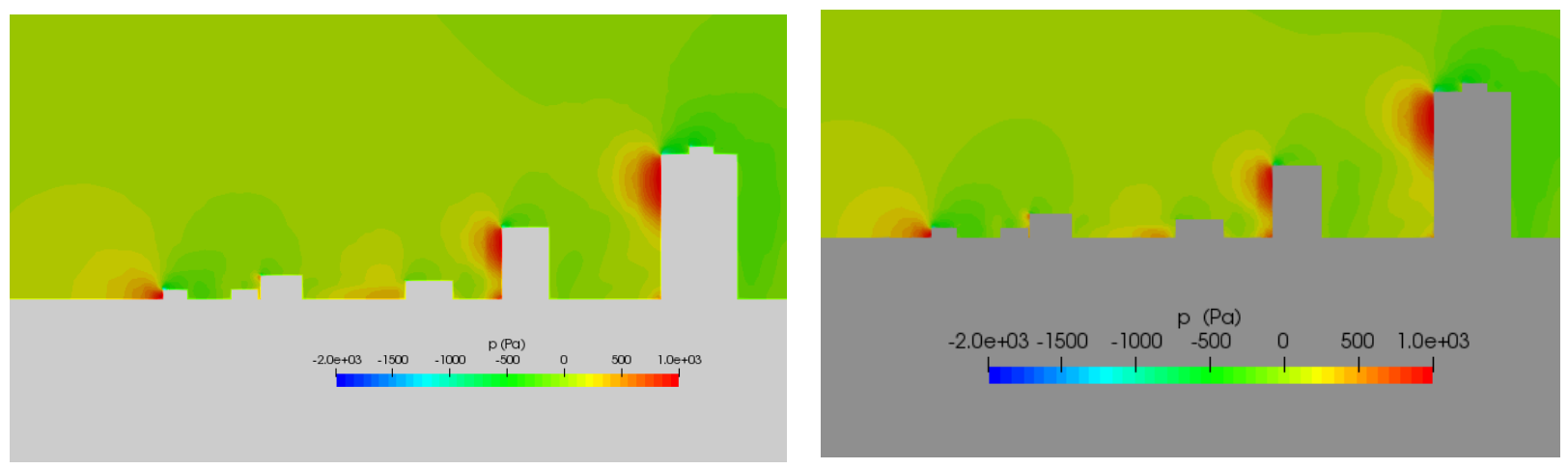

(b) Pressure
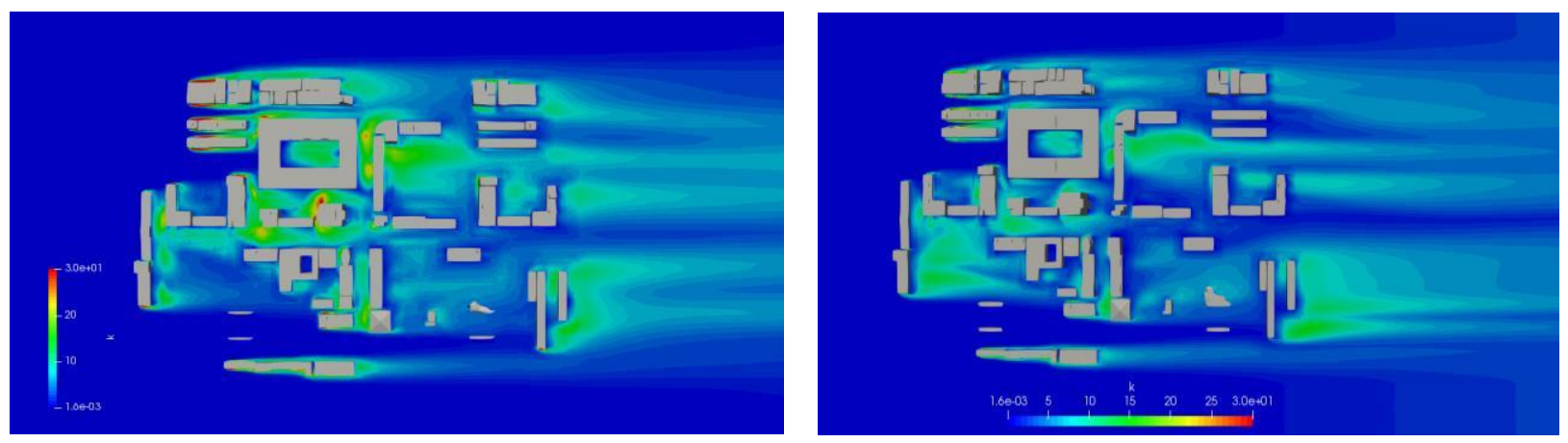

(c) Kinetic energy at $2.5 \mathrm{~m}$ above the ground (plan view)

Figure 7: The comparison of flow field in Y-direction for both RNG k-epsilon model (left side) and SST k-omega model (right side).

\section{Conclusion}

In this study, an idealized urban area was generated to investigate the use of different turbulence modeling approaches such as the RNG k-epsilon and k-omega models to simulate the wind flow at different wind directions. The results showed that the predicted pressure and velocity profiles obtained from both modeling approaches are identical, whereas significant difference can be seen in the kinetic energy up to $40 \mathrm{~m}$ height; the k-epsilon model predicts higher kinetic energy than the k-omega model.Based on the outcome results, it can be concluded that using two or more turbulence models can be more efficient in reducing the numerical errors and overcome the effects of utilizing only single numerical simulation in the field of aerodynamic engineering.

\section{References}

A.K. Roy and P.K. Bhargava. (2012). CFD Modelling of Wind Flow around Buildings for Wind Energy Conversion. National Conference on Emerging trends of energy conservation in buildings, (pp. 370-379). Roorkee, India.

ASCE . (2013). Minimum design loads for building and other structures.

WASHINGTON, D.C.: ASCE (American Society of Civil Engineers). 
Hao Wang, Jiaojiao Ding, Bing Ma, Shuaibin Li. (2014, Aauugust 15). Aerodynamic simulation of wind turbine blade airfoil with different turbulence models. Journal of Vibroengineering, 16(5), 2474-2483.

Howard H. Hu. (2012). Chapter 10 - Computational Fluid Dynamics. Waltham, MA 02451, USA: Elsevier.

Lee D. (1993). Application of computational fluid dynamics in transonic aerodynamic design. American Institute of Aeronautics and Astronautics, Aerospace Sciences Meeting and Exhibition.

Menter, F. R. (1993). Zonal Two Equation k- $\omega$ Turbulence Models for Aerodynamic Flows. AIAA Journal.

Ping He, Tadahisa Katayama, Tetsuo Hayashi, Jun-ichiro Tsutsumi. (1997).

Numerical simulation of air flow in an urban area with regularly aligned blocks. Journal of Wind Engineering, 281-291.

S.Reiter. (2008). Validation Process for CFD Simulations of Wind Around Buildings. European Built Environment CAE Conference. Belgium: University of Liège.

Yakhot, V., Orszag, S.A., Thangam, S., Gatski, T.B. \& Speziale, C.G. (1992). Development of turbulence models for shear flows by a double expansion technique. Physics of Fluids A, 4(7), 1510-1520. 